\title{
Decreased calcineurin immunoreactivity in the postmortem brain of a patient with schizophrenia who had been prescribed the calcineurin inhibitor, tacrolimus, for leukemia
}

\author{
This article was published in the following Dove Press journal: \\ Neuropsychiatric Disease and Treatment \\ 6 July 2016 \\ Number of times this article has been viewed
}

\author{
Akira Wada ${ }^{1,2}$ \\ Yasuto Kunii' \\ Jyunya Matsumoto' \\ Mizuki Hino' \\ Atsuko Nagaoka' \\ Shin-ichi Niwa ${ }^{3}$ \\ Hirooki Yabe' \\ 'Department of Neuropsychiatry, \\ Fukushima Medical University \\ School of Medicine, Fukushima \\ City, Fukushima, ${ }^{2}$ Department of \\ Neuropsychiatry, The University of \\ Tokyo Hospital, Bunkyo-ku, Tokyo, \\ ${ }^{3}$ Department of Psychiatry, Aizu \\ Medical Center, Fukushima Medical \\ University, Aizuwakamatsu City, \\ Fukushima, Japan
}

Background: The calcineurin $(\mathrm{CaN})$ inhibitor, tacrolimus, is widely used in patients undergoing allogeneic organ transplantation and in those with certain allergic diseases. Recently, several reports have suggested that $\mathrm{CaN}$ is also associated with schizophrenia. However, little data are currently available on the direct effect of tacrolimus on the human brain.

Case: A 23-year-old Japanese female experienced severe delusion of persecution, delusional mood, suspiciousness, aggression, and excitement. She visited our hospital and was diagnosed with schizophrenia. When she was 27 years old, she had severe general fatigue, persistent fever, systemic joint pain, gingival bleeding, and breathlessness and was diagnosed with acute myelomonocytic leukemia. Later she underwent bone marrow transplantation (BMT), she was administered methotrexate and cyclosporin A to prevent graft versus host disease (GVHD). Three weeks after BMT, she showed initial symptoms of GVHD and was prescribed tacrolimus instead of cyclosporin A. Seven months after BMT at the age of 31 years, she died of progression of GVHD. Pathological anatomy was examined after her death, including immunohistochemical analysis of her brain using anti-CaN antibodies. For comparison, we used our previous data from both a schizophrenia group and a healthy control group. No significant differences were observed in the percentage of CaN-immunoreactive neurons among the schizophrenia group, healthy control group, and the tacrolimus case (all $P>0.5$, analysis of covariance). Compared with the healthy control group and schizophrenia group, the percentages of $\mathrm{CaN}$-immunoreactive neurons in layers III-VI of the BA46 and the putamen tended to be lower in the tacrolimus case.

Conclusion: Tacrolimus may decrease $\mathrm{CaN}$ immunoreactivity in some regions of the human brain. Thus, tacrolimus may introduce side effects such as cognitive dysfunction and extrapyramidal symptoms. In addition, we also found that the effect of tacrolimus on $\mathrm{CaN}$ immunoreactivity in human brain was stronger than the effect of schizophrenia.

Keywords: calcineurin, calcineurin inhibitors, schizophrenia, postmortem brain, immunohistochemistry

\section{Introduction}

The calcineurin $(\mathrm{CaN})$ inhibitor, tacrolimus, is a nonsteroidal, anti-inflammatory immunosuppressive drug mainly used to treat patients undergoing allogeneic organ transplantation and those with atopic dermatitis. ${ }^{1} \mathrm{CaN}$ is a heterodimeric calciumdependent serine/threonine protein phosphatase, consisting of a catalytic ( $\mathrm{CaN} \mathrm{A}$ ) and a regulatory ( $\mathrm{CaN} \mathrm{B})$ subunits, and plays a critical role in cellular responses and calcium signaling. ${ }^{2}$ Tacrolimus induces not only mild neurological side effects such
Department of Neuropsychiatry

Fukushima Medical University School of Medicine, I Hikarigaoka, Fukushima City, Fukushima 960- I295, Japan

Tel +8| 24547 |33|

Fax +8I 245486735

Email nishityo4nishihara@gmail.com 
as tremor but also psychiatric side effects such as manic-like psychosis and relapse of schizophrenia. ${ }^{3,4}$ On the other hand, several reports suggested that $\mathrm{CaN}$ dysfunction may be a risk factor for schizophrenia. Forebrain-specific PPP $3 C C$ (encodes the CaN A $\gamma$-catalytic subunit) knockout mice display multiple abnormal activities related to schizophrenia, such as increased locomotor activity, decreased social interaction, impairments in prepulse inhibition, and latent inhibition. ${ }^{5,6}$ Another report showed decreased CaN A protein levels and decreased mRNA of the three subunits of $\mathrm{CaN}$ A in the hippocampus of schizophrenia patients. ${ }^{7}$

Chang et $\mathrm{al}^{8}$ reported on the B-cell lymphoma-2 (Bcl2)-CaN-dopamine- and cAMP-regulated phosphoprotein of $32 \mathrm{kDa}$ (DARPP-32) feedback mechanism in regulating serine 1755 phosphorylation and apoptosis in primary human chronic lymphocytic leukemia cells. However, there were no previous reports that acute myelomonocytic leukemia (AML) itself has direct influence on $\mathrm{CaN}$ in the brain.

To investigate the effect of tacrolimus in the brain, we examined the immunoreactivity of $\mathrm{CaN}$ using immunohistochemistry in the dorsolateral prefrontal cortex, hippocampus, caudate, and putamen of a postmortem brain of a patient with schizophrenia who had been treated with tacrolimus. As healthy and disease controls, we used our previously reported data. ${ }^{9}$ There were no previous reports of AML itself having a direct influence on $\mathrm{CaN}$ in the brain. If we only aimed to investigate the effect of tacrolimus on the brain, we would have focused on characterizing the effect of tacrolimus in AML patients without prior history of schizophrenia. We aimed to investigate whether tacrolimus treatment or acquiring schizophrenia had a stronger effect on the $\mathrm{CaN}$ immunoreactivity in the brain.

\section{Case report}

A 23-year-old Japanese female visited a local psychiatric clinic in May 1990 due to nonspecific physical symptoms. She had poor premorbid functioning, and her mother had been diagnosed with schizophrenia. She had no history of neurological disorders or substance abuse. She first received a diagnosis of psychogenic reaction. Several months after she first visited that clinic, she experienced severe delusion of persecution, delusional mood, suspiciousness, aggression, and excitement. She first visited our hospital in November 1992 and was diagnosed with schizophrenia. She was prescribed antipsychotics, but she initially refused them. She and her mother had "shared psychotic disorder". Because they shared the same delusion of persecution, they moved together many times to various places based on delusions. In November 1992, she began to take haloperidol (4.5 mg/d) and chlorpromazine $(200 \mathrm{mg} / \mathrm{d})$ for her psychotic symptoms. Five months after starting antipsychotics, her psychotic symptoms had improved.

In June 1996, she experienced severe general fatigue, persistent fever $\left(\geq 38^{\circ} \mathrm{C}\right)$, systemic joint pain, gingival bleeding, and breathlessness. Her blood tests showed pancytopenia (white blood cells, $3.1 \times 10^{3} / \mathrm{mm}^{2}$; red blood cells, $2.6 \times 10^{6} / \mu \mathrm{L}$; hemoglobin, $7.5 \mathrm{~g} / \mathrm{dL}$; and platelets, $8.8 \times 10^{4} / \mu \mathrm{L}$ ) and the presence of blast cells. We consulted a hematologist, and she was diagnosed with AML. In September 1996, she underwent bone marrow transplantation (BMT). After BMT, she was administered methotrexate and cyclosporin A to prevent graft versus host disease (GVHD). At the same time, we stopped prescribing antipsychotics. Three weeks after BMT, she had diarrhea and systemic eruption. We judged that she had initial symptoms of GVHD, and the hematologist prescribed tacrolimus instead of cyclosporine A. In December 1996, her auditory hallucination, delusions, and excitement recurred. We needed to prescribe risperidone so that she could continue her treatment safely. Her general condition gradually worsened. In February 1997, she experienced disturbance of consciousness, hypotension, and respiratory failure. In April 1997, she died of progression of GVHD at the age of 31 years.

Pathological anatomy was examined after her death. The use of postmortem human brain tissues for the present study was approved by the Ethics Committee of Fukushima Medical University (FMU) and complied with the Declaration of Helsinki. All procedures were carried out with the informed written consent of the next of kin. The postmortem interval was 9.5 hours. Her brain weight was 1,232 $\mathrm{g}$ with no indication of gross neurological pathology. Brain tissue blocks were obtained from the right hemisphere of the dorsolateral prefrontal cortex (BA46), hippocampus, caudate, and putamen and fixed in 10\% formalin, embedded in paraffin, and sliced into $5 \mu \mathrm{m}$ sections. We obtained ten sections and used one section in this analysis. Immunohistochemistry was performed in accordance with our previous protocol..$^{9-11}$ The sections were deparaffinized in xylene and rehydrated through a decreasing alcohol series $(100 \%, 90 \%, 80 \%$, and $70 \%$ ethanol). Endogenous peroxidase activity was quenched by incubating in $0.3 \%$ hydrogen peroxide in methanol at room temperature for 20 minutes. The sections were washed with phosphate-buffered saline (PBS: 0.01 M sodium phosphate, $\mathrm{pH} 7.3$ ), microwaved for 15 minutes in $10 \mathrm{mM}$ citrate buffer for antigen recovery, and washed with PBS. The sections were incubated in 5\% skim milk in PBS for 30 minutes at room temperature to reduce nonspecific binding, incubated overnight at $4^{\circ} \mathrm{C}$ in rabbit polyclonal antibodies against 
human CaN (1717-0909; AbD Serotec, Oxford, UK, 1:50), which recognizes the $60 \mathrm{kDa}$ mammalian $\mathrm{CaN}$ catalytic subunit (CaN A), in PBS, and washed in PBS three times for 5 minutes each. We used normal rabbit serum instead of the primary antibody as a negative control. The sections were incubated with the second antibody (biotinylated anti-rabbit IgG; Nichirei Corporation, Tokyo, Japan) for 20 minutes at room temperature and washed in PBS three times for $5 \mathrm{~min}$ utes each. Subsequently, the sections were incubated with peroxidase-labeled streptavidin for 15 minutes at room temperature and washed in PBS three times for 5 minutes each. The sections were treated with diaminobenzidine (Wako Pure Chemical Industries, Ltd., Osaka, Japan) for 5 minutes at room temperature to visualize the immunoreaction products and then washed with PBS to terminate the reaction. For nuclear counterstaining, the sections were treated with Carracci's hematoxylin for 20 seconds. CaN-immunoreactive (CaN-IR) cells were examined under a microscope equipped with a digital camera (Olympus BX51; Olympus Corporation, Tokyo, Japan) at 100× magnification, and parenchymal cell numbers were counted using WinROOF Version 5.5 (Mitani Corporation, Tokyo, Japan). Neurons and neuroglial cells were distinguished by morphology, nuclear size, nuclear membrane thickness, and chromatin homogeneity. ${ }^{10-12} \mathrm{We}$ defined neurons in which staining was more intense than background as $\mathrm{CaN}-\mathrm{IR}$. Image acquisition was performed to show the representative staining pattern of each region and to include approximately the same number of total neurons. In the BA46, three images were randomly selected from each of layers II-VI. The mean percentage of CaN-IR neurons (number of $\mathrm{CaN}-\mathrm{IR}$ neurons/number of total neurons) was calculated for each layer. In the hippocampus, the CA1, CA2, $\mathrm{CA} 3$, and CA4 subfields and dentate gyrus were investigated by randomly selecting three, one, one, three, and two images, respectively. The caudate and putamen were analyzed separately by randomly selecting three images each. A single researcher blinded to the tissue source was responsible for all cell counting and data analyses.

The percentages of CaN-IR neurons in the BA46, hippocampus, caudate nucleus, and putamen are shown in Figure $1 \mathrm{~A}-\mathrm{C}$. To compare this patient with a schizophrenia group and healthy control group, we used previously reported data. ${ }^{9}$ No significant differences were observed in the percentage of CaN-IR neurons among the schizophrenia group, healthy control group, and the tacrolimus case (all $P>0.5$, analysis of covariance). Compared with the healthy control group and schizophrenia group, the percentages of CaN-IR neurons in layers III-VI of the BA46 and the putamen tended to be lower in the tacrolimus case (Figure 1A and C). Representative images of CaN-IR neurons in the prefrontal cortex (BA46, layers $\mathrm{V}-\mathrm{VI})$ are shown in Figure 2.

First, we considered the possibility of an association between our patient's psychiatric symptoms and tacrolimus. The percentage of CaN-IR neurons in layers III-VI of the BA46 tended to be lower in patients with schizophrenia compared with the healthy control group. Furthermore, the percentage of CaN-IR neurons was even lower in the tacrolimus patient compared with the schizophrenia alone group. Cognitive dysfunction due to hypofunction of the frontal lobe is well known in schizophrenia. ${ }^{13}$ In addition, a side effect of tacrolimus is delirium, and this form of cognitive dysfunction is more severe than schizophrenia without delirium. Thus, the decrease in $\mathrm{CaN}$ immunoreactivity in the frontal lobe may reflect cognitive dysfunction.

Next, we considered the results from the caudate and putamen. The percentage of CaN-IR neurons in the putamen tended to be higher in patients with schizophrenia compared with healthy subjects, but the percentage of CaN-IR neurons was lower in the tacrolimus case than in the schizophrenia group. Tacrolimus may cause side effects of tremor and dysarthria, which occur when a patient has dysfunction of the striatum. Lower immunoreactivity of $\mathrm{CaN}$ induced by tacrolimus in the striatum may mediate these extrapyramidal symptoms.

Lee et $\mathrm{al}^{14}$ reported that tacrolimus reduces nuclear expression of $\mathrm{CaN}$ in a renal ischemia-reperfusion injury mouse model. They suggested that tacrolimus decreases $\mathrm{CaN}$ expression both in the cytoplasm and in the nucleus. Our data indicated that the percentages of CaN-IR neurons in layers III-VI of the BA46 and putamen tended to be lower in the tacrolimus case compared with the schizophrenia group. As in the report by Lee et al, tacrolimus may decrease the expression of $\mathrm{CaN}$ in some regions of the human brain. Overall, when we considered the results from the frontal, caudate, and putamen together, we found that the effect of tacrolimus in the human brain may be stronger than the effect of schizophrenia.

Eastwood et $\mathrm{al}^{7}$ reported decreased CaN A protein levels and decreased mRNA of the three subunits of CaN A in the hippocampus of schizophrenia patients. This report does not support our findings in the hippocampus. This discrepancy could be attributed to the small sample numbers in our study.

Our study has a number of limitations. Only one tacrolimus case was included in this report. The effect of tacrolimus on the human brain might be subject to intersubject differences in $\mathrm{CaN}$ immunoreactivity and thus generalization of these findings might be difficult. In addition, the lack of a 

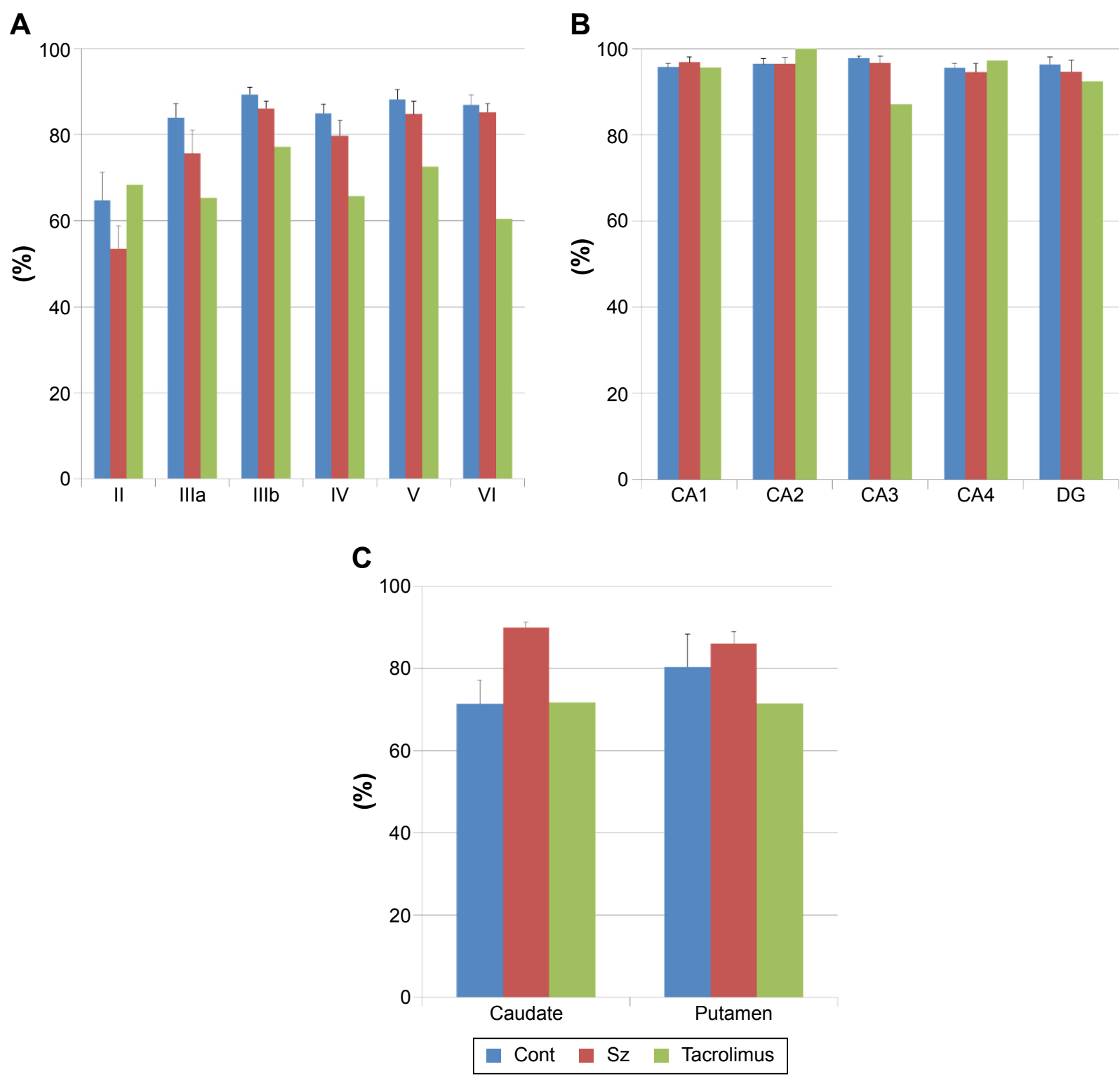

Figure I Quantitative analysis of CaN-IR neurons: layers II-VI of the prefrontal cortex (BA46), hippocampus, caudate nucleus, and putamen.

Notes: In each histogram, "Sz" indicates the schizophrenia group, "cont" indicates the control group, and "tacrolimus" indicates the tacrolimus case. Analysis of covariance was used to compare the percentage of $\mathrm{CaN}$-IR neurons among the three groups. (A) Percentage of CaN-IR neurons in each layer of the prefrontal cortex (BA46). (B) Percentage of CaN-IR neurons in each subfield of the hippocampus. (C) Percentage of CaN-IR neurons in the caudate and putamen.

Abbreviations: $\mathrm{CaN}-\mathrm{IR}$, calcineurin-immunoreactive; DG, dentate gyrus.

control group (patients with leukemia but without tacrolimus treatment and schizophrenia, patients with leukemia treated using tacrolimus but without schizophrenia) makes drawing firm conclusion regarding efficacy difficult. It is also possible that the AML might have directly influenced $\mathrm{CaN}$ immunoreactivity in the brain. Further studies using a larger sample size are warranted to investigate $\mathrm{CaN}$ immunoreactivity in the postmortem brains of leukemia patients without tacrolimus treatment and schizophrenia, leukemia patients treated with tacrolimus but without schizophrenia, and schizophrenia patients with tacrolimus-treated AML. Second, this research was conducted using immunohistochemical analysis only. Further studies using other methods, such as in situ hybridization, are warranted. Third, CaN-IR neurons were defined as having greater staining intensity compared to background. This method allows for ambiguity because it is dependent on human assessment. In this context, the use of in situ hybridization for future studies is warranted. Finally, if the patient carried the PPP $3 C C$ SNP, the level of CaN might have already been low, instead of attributable to the tacrolimus treatment. However, we could not obtain the SNPs data because we were unable to use frozen brain 

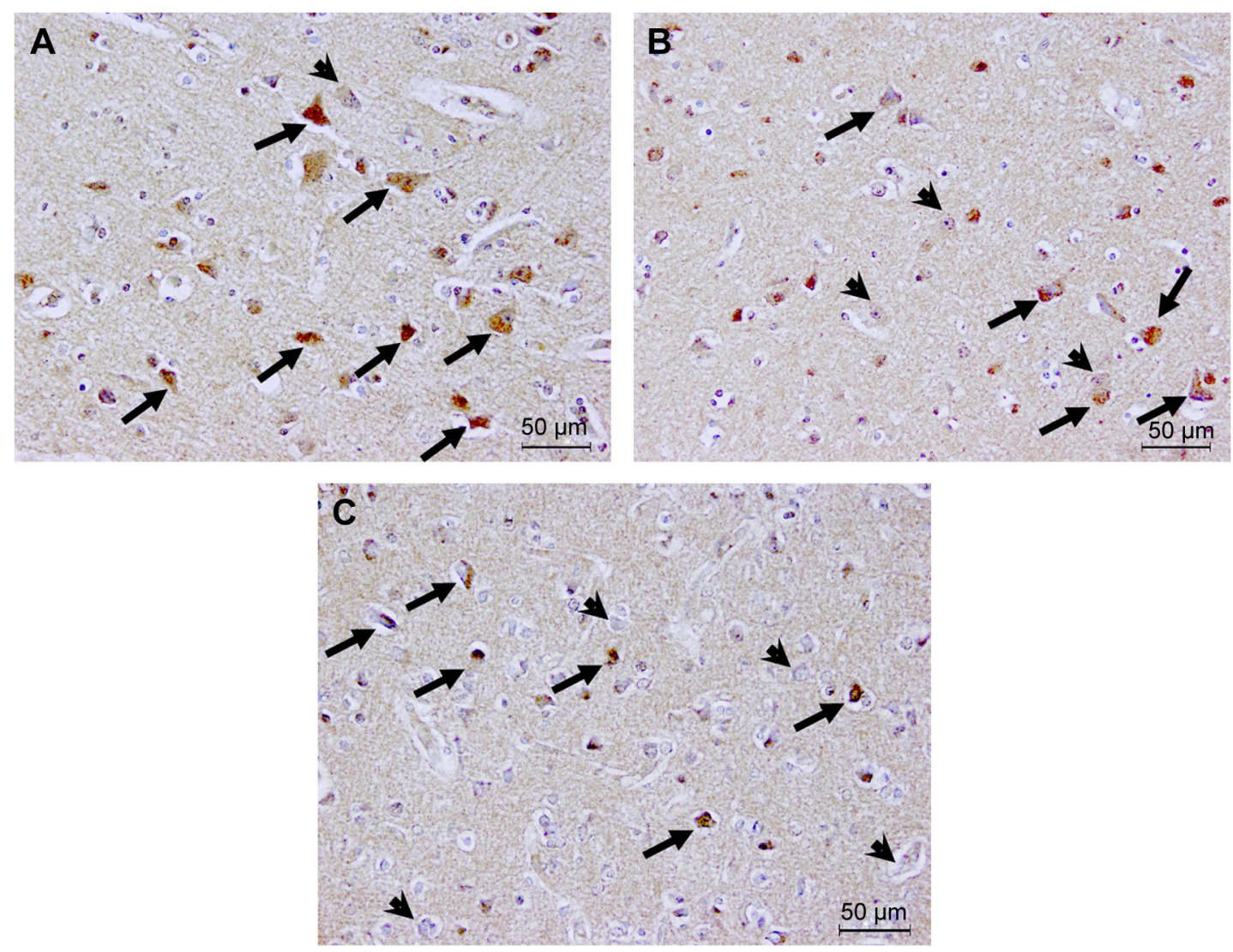

Figure $2 \mathrm{CaN}-\mathrm{IR}$ neurons in brain sections from controls, patients with schizophrenia, and a patient with both schizophrenia and acute myelomonocytic leukemia treated with tacrolimus.

Notes: (A) Calcineurin-immunoreactive ( $\mathrm{CaN}$-IR) cells in the prefrontal cortex (BA46, layers $\mathrm{V}-\mathrm{VI})$ of a control brain (72-year-old male with a postmortem interval [PMI] of I2.5 hours). (B) CaN-IR cells in the prefrontal cortex (BA46, layers $\mathrm{V}-\mathrm{VI}$ ) of the brain of a patient with schizophrenia (48-year-old male with a PMI of I0 hours). (C) CaN-IR cells in prefrontal cortex (BA46, layers $\mathrm{V}-\mathrm{VI})$ in the brain of a patient with both schizophrenia and acute myelomonocytic leukemia treated with tacrolimus (3I-year-old female with a PMI of 9.5 hours). Immunopositive neurons are indicated by arrows, and immunonegative neurons are indicated by arrowheads. Magnification $\times 200$.

samples in the tacrolimus case. Further studies are needed to obtain the SNPs data from frozen samples.

\section{Conclusion}

We performed immunohistochemical analysis using human brain tissue from a patient with schizophrenia and AML treated with tacrolimus. Our data suggested that tacrolimus may decrease the immunoreactivity of $\mathrm{CaN}$ in some regions of the human brain. In addition, the percentage of CaN-IR neurons in layers III-VI of the BA46, caudate, and putamen tended to be lower in patients with schizophrenia complicated by tacrolimus-treated AML compared to the schizophrenia group. These data also suggested that the effect of tacrolimus in the human brain was stronger than the effect of schizophrenia.

\section{Acknowledgments}

We thank Hiromi Onuma for her assistance with coordinating tissue donations. We also thank the families of the deceased for the donations of brain tissue and the time and effort devoted to the consent process and interviews.

\section{Disclosure}

The authors report no conflicts of interest in this work.

\section{References}

1. Miyata S, Ohkubo Y, Mutoh S. A review of the action of tacrolimus (FK506) on experimental models of rheumatoid arthritis. Inflamm Res. 2005;54(1):1-9.

2. Rusnak F, Mertz P. Calcineurin: form and function. Physiol Rev. 2000; 80(4):1483-1521

3. Bersani G, Marino P, Valeriani G, et al. Manic-like psychosis associated with elevated trough tacrolimus blood concentrations 17 years after kidney transplant. Case Rep Psychiatry. 2013;2013:926395.

4. Lin Y, Sun IW, Liu SI, Loh el-W, Lin YC. Tacrolimus ointment-induced relapse of schizophrenia: a case report. Int $J$ Neuropsychopharmacol. 2007;10(6):851-854.

5. Miyakawa T, Leiter LM, Gerber DJ, et al. Conditional calcineurin knockout mice exhibit multiple abnormal behaviors related to schizophrenia. Proc Natl Acad Sci US A. 2003;100(15):8987-8992.

6. Zeng H, Chattarji S, Barbarosie M, et al. Forebrain-specific calcineurin knockout selectively impairs bidirectional synaptic plasticity and working/episodic-like memory. Cell. 2001;107(5):617-629. 
7. Eastwood SL, Burnet PW, Harrison PJ. Decreased hippocampal expression of the susceptibility gene PPP3CC and other calcineurin subunits in schizophrenia. Biol Psychiatry. 2005;57(7):702-710.

8. Chang MJ, Zhong F, Lavik AR, Parys JB, Berridge MJ, Distelhorst CW. Feedback regulation mediated by Bcl-2 and DARPP-32 regulates inositol 1,4,5-trisphosphate receptor phosphorylation and promotes cell survival. Proc Natl Acad Sci U S A. 2014;111(3):1186-1191.

9. Wada A, Kunii Y, Ikemoto K, et al. Increased ratio of calcineurin immunoreactive neurons in the caudate nucleus of patients with schizophrenia. Prog Neuropsychopharmacol Biol Psychiatry. 2012;37(1):8-14.

10. Kunii Y, Ikemoto K, Wada A, et al. Detailed DARPP-32 expression profiles in postmortem brains from patients with schizophrenia: an immunohistochemical study. Med Mol Morphol. 2011;44(4):190-199.

11. Kunii Y, Yabe H, Wada A, Yang Q, Nishiura K, Niwa S. Altered DARPP-32 expression in the superior temporal gyrus in schizophrenia. Prog Neuropsychopharmacol Biol Psychiatry. 2011;35(4): $1139-1143$.
12. Selemon LD, Rajkowska G, Goldman-Rakic PS. Abnormally high neuronal density in the schizophrenic cortex. A morphometric analysis of prefrontal area 9 and occipital area 17. Arch Gen Psychiatry. 1995; 52(10):805-818. discussion 819-820.

13. Yoon JH, Minzenberg MJ, Ursu S, et al. Association of dorsolateral prefrontal cortex dysfunction with disrupted coordinated brain activity in schizophrenia: relationship with impaired cognition, behavioral disorganization, and global function. Am J Psychiatry. 2008;165(8): 1006-1014.

14. Lee SH, Choi J, Kim H, et al. FK506 reduces calpain-regulated calcineurin activity in both the cytoplasm and the nucleus. Anat Cell Biol. 2014;47(2):91-100.

\section{Publish your work in this journal}

Neuropsychiatric Disease and Treatment is an international, peerreviewed journal of clinical therapeutics and pharmacology focusing on concise rapid reporting of clinical or pre-clinical studies on a range of neuropsychiatric and neurological disorders. This journal is indexed on PubMed Central, the 'PsycINFO' database and CAS, and is the official journal of The International Neuropsychiatric Association (INA). The manuscript management system is completely online and includes a very quick and fair peer-review system, which is all easy to use. Visit http://www.dovepress.com/testimonials.php to read real quotes from published authors.

Submit your manuscript here: http://www.dovepress.com/neuropsychiatric-disease-and-treatment-journal 